\title{
Performance and Capacity Planning of NGN
}

\author{
Mustafa Shakir, Mahmood Ashraf Khan, Shahzad A. Malik, Izhar ul Haq \\ Department of Electrical Engineering, COMSATS Institute of Information Technology, Islamabad, Pakistan \\ E-mail: mustafa.shakir@comsats.edu.pk \\ Received September 12, 2011; revised October 14, 2011; accepted October 22, 2011
}

\begin{abstract}
There have been various emerging trends in the deployment of telecom networks recently. Due to liberalization and technological advancements a common core network for voice and data based on MPLS has been implemented by Telecom service providers. By offering a variety of data and multimedia services, the revenue generating process can also be maximized. The economic and regulation trends also have to be synchronized for the proper and step by step replacement of traditional network by NGN. The current flow is to provide users with multitude of services thus meeting their high demands and enhancing revenues for network operators. Class 5 and Class 4 are our legacy circuit switches based upon TDM to transport voice. For handling control functions, call setup and teardown SS7 signaling was used. The traditional network was designed to support voice services only not being able to meet customers' demands who require a variety of services at an optimum cost. The aim of this work is to present the role of NGN encompassing the telecom network, architecture and technologies. Also it has emerged as a new platform to decrease the CAPEX and OPEX of overall network infrastructure and to maximize their revenues. In this paper, we present an upcoming architecture for the transition to NGN which is future proof for going through the migration phase along with the capacity and planning of our network.
\end{abstract}

Keywords: Next Generation Network, Convergence, MSAN, Softswitch, FTTH

\section{Introduction}

There is a changed trend of adoption of packet switched networks from circuit switched networks. The scope of services to be provided to the users can be widened thus availability of anytime anywhere access for satisfying customers and meeting the enhanced demands [1]. Most service operators are targeting NGN in view of market demands and IP would be the core network.

The ITU defines the Next Generation Network as a packet based network able to provide services and make use of multiple broadband, QoS enabled transport technologies in which service related functions are independent of underlying transport related technologies [2]. The access is common to all and different network operators are integrated at the access layer. NGN also provides ubiquitousness and mobility to its subscribers. NGN has a hierarchical four layered architecture which due to its nature can be able to support additional services. It is a packet based network which provides efficient networking features in addition to supporting new services through open interfaces. NGN also has separate application and network layers thus providing the room to further improve services. Third parties can also contribute to development of application applications for subscribers. With transition to IP networks the average per line costs of increasing networks could increase leading to deteriorated QoS. In Section 2, we would go through the existing trends followed by service providers. In Section 3 the implementation of MSAN and switch up gradation is described. Capacity planning amongst the NGN elements is discussed in Section 4. Finally we conclude our observations in Section 5.

\section{Switching Approach by Network Operators}

The telecom service providers have to take into consideration the high expectations such as Broadband and Multimedia services for customers' satisfaction. The equipments for switching and access would have to be upgraded and replaced so as to promote the convergence trend. The standardization bodies throughout the world have worked on the protocols and general architecture for the advanced networking aspects in new approach. The new architecture would support not only existing 
telecom infrastructures but would also ensure a smooth transition to an ip based network. British Telecom and SingTel are adopting the converged approach thus implementing a single multi-service network [3]. Various operators are working upon implementation of NGN in their core networks. The operators would pursue a phased approach towards upgrading to the corresponding NGN components while providing the services of traditional network at the same time. The NGN setup is shown in Figure 1. The softswitch (SS) provides call control services as well as Media Gateway control since it handles many switching functions and has replaced the traditional switch. The Trunk Gateway (TGW) and Access Gateway (AGW) are controlled by the softswitch and act as the interface between circuit and packet switched networks converting ip to TDM traffic and TDM traffic to ip. The Application Server (AS) provides enhanced features which are not available in the softswitch. A protocol used to control call setup, connection and termination. The call control protocols used in softswitch are SS7 for switched circuit network, whereas SIP and H.323 are implemented for call control in packet switched networks. The softswitch controls media gateways by means of MGCP. The SIGTRAN (Signaling Transport) protocol carries SS7 signals over the internet. The immediate and one step conversion of existing networks to NGN would not be possible so it has to be exe- cuted in a proper way. At the core IP network shown in Figure, IP based components would be implemented which would realize a MPLS approach and multimedia services would have to be provided thus achieving our targets of Fixed Mobile Convergence [4].

The network operators divide their traditional infrastructure into regions or domains as per the requirements of operation and maintenance, traffic engineering and distribution and the buildup of sites in various areas. The network can be seen as comprising of a number of domains while we realize the NGN solution for switching i.e. the Soft Switch. Our IP based network at the core would be able to provide a better QoS and processing a capability to handle a large number of subscribers. The softswitch serves as the central device in the telecommunications system. The call agent in the soft switch carries out functions of signaling, billing and services. The Media Gateway which is controlled by the softswitch acts as the interface between circuit and packet switched networks converting ip to TDM traffic and TDM traffic to ip. The Signaling Gateway converts PSTN signaling between TDM bearer mode and IP packets mode and is applied on the access layer of NGN thus can process both SIGTRAN and SS7 protocols keeping ahead in signaling link density, capacity and signaling processing capacity. The access gateway is the line interface to the core ip network and connects subscribers with analog

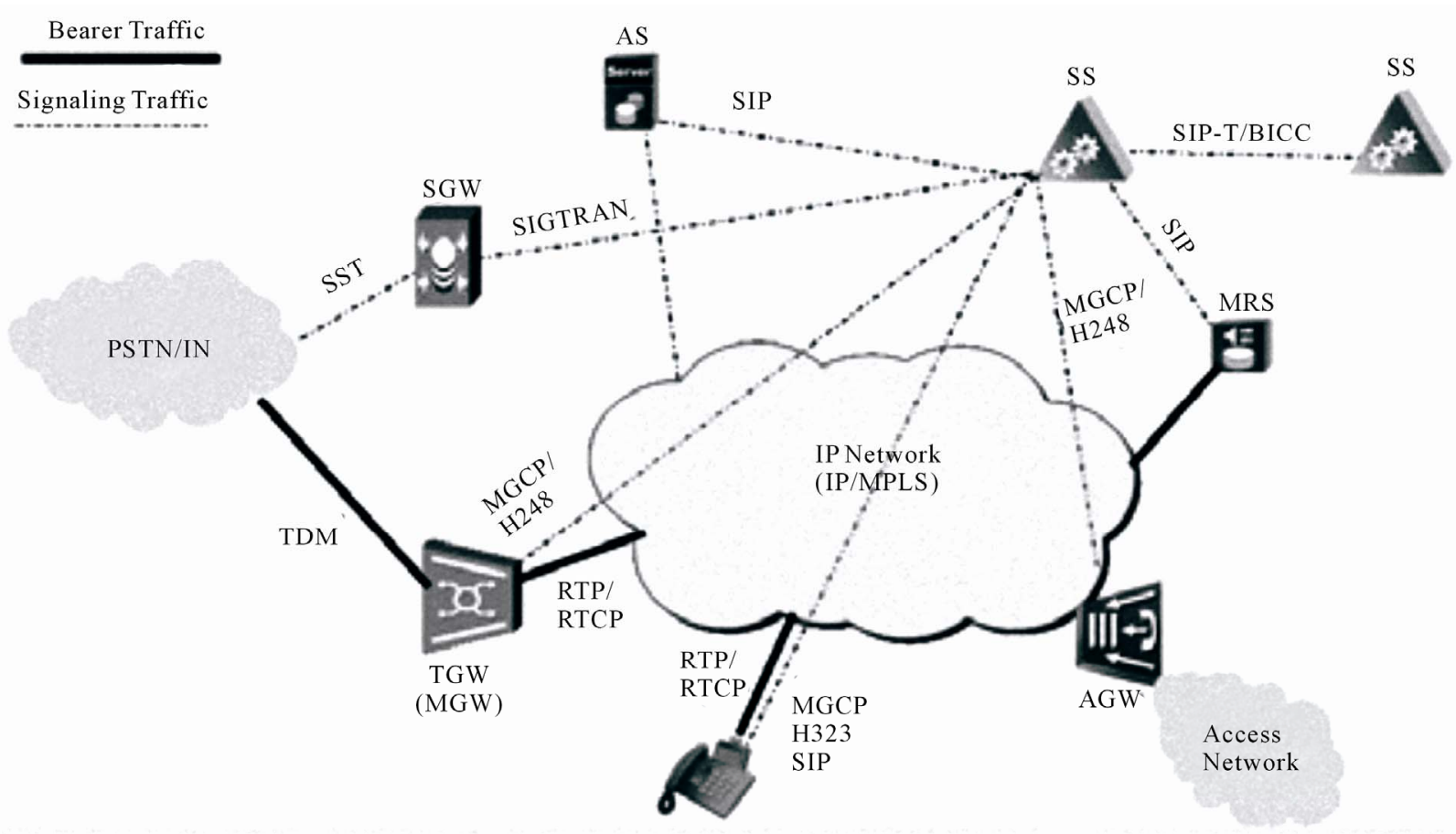

SGW = Signaling Gateway, AS = Application Server, SS = Softswitch, MRS = Media Resource Server, TGW $=$ Trunking Gateway, $\mathrm{AGW}=$ Access Gateway

Figure 1. NGN approach for switching. 
subscriber access, ISDN and V5 subscriber access. The service logics and individual service features are implemented on the application server. Interconnection between Application Server and Media Gateway Controller is realized using SIP protocol. The nature of NGN as a result of division into layers provides flexibility, scalability and openness of the converged NGN platform.

\section{Multi Service Access Nodes: The Gateways to NGN}

The deployment of networks by different operators would more or less evolve on the same extent as access technologies would be the same. The network at the core level would be ATM/SDH which is used to interconnect the Class 5 switches to the Class 4 switches of higher order [5]. This ATM network serves as source of backhaul broadband services from DSLAMs located at the CO to ISPs and gateways. The migration to NGN would be achieved by converting the core network to IP. The equipment for NGN would replace the legacy switches as a next step.

Broadband applications would be provided through FTTH and giving a bandwidth of several Gbps. So the service providers are upgrading their networks by deploying the MSAN. British Telecom, France Telecom, Telekon Austria and Belgacom are upgrading their networks. The British Telecom has invested a huge amount of up to $\$ 17$ billion to upgrade their existing networks. In Asia, NTT has developed its own Next Generation Network thus providing optical fiber based broadband access which would deliver a variety of broadband ubiquitous services. SingTel is adopting the path towards convergence by combining $3 \mathrm{G}$ and video services across mobile, fixed-line and broadband platforms.

\subsection{Switch Upgradation}

The change in the infrastructure to move on to the new switching systems would be phase wise thus capacity requirements could be greater and OSS would be accommodating the new IP infrastructure and data from traditional legacy systems have to be transferred and transformed to a new OSS. The BSS (Business Support System) migration will define the operation and functioning according to the new NGN IP network.

For the new scenario Class 5 switches and DSLAMs would be replaced by MSAN equipment (equipped with POTS, ISDN and DSLAM cards) and these MSANs would require IP over fiber connections to the core network.

The MSAN cards convert xDSL, POTS and other services to IP. Shifting the core networks to IP is an exten- sive job which would take several years and with new DSLAM installation these would have to be IP based so that compatibility can be ensured.

\subsection{Using MSAN}

The new strategy would be to install MSANs with DSLAM cards and with the completion of IP core POTS and other cards can be added to avoid the need for total replacement.

MSANs can be fully operational with the all-IP core when Softswitch is used for call set up and authentication functions. MSAN would be thus used to provide the broadband services while other services such as POTS would be provided over copper. When the IP core is complete customer lines would need to be transferred from copper pairs onto MSAN ports. VoIP would be prevalent as voice, video and data can all be provided as Triple play application.

\subsection{MSAN in the Future}

Service providers are opting for FTTH (Fiber to the Home) in the long term. The step involved in migrating to FTTH is to convert from active MSANs to passive Fibre distribution hubs. For each node's service coverage area a fiber dig would be needed from each home and back to node.

There are two options for FTTH/P, the first one being a passive optical network (PON) using shared fibres, which are more cost-effective for long $\mathrm{CO}$ to node distances such as in rural locations, or a point-to-point (P2P) for metro and urban situations, where every customer has their own fibre back to the IP-core. Most operators will need a mix of both.

In case of PON, the MSAN will be replaced with a fibre distribution hub where passive optical splitters combine the light wave signals from 32, 64 or 128 customers on to a fibre pair back to the IP-core. Alongside this an optical fibre cross-connection or patching frame (similar to that we currently have for copper) to provide moves, adds and changes (MAC) flexibility. For P2P, a lot of new fibres back to the IP-core need to be blown-in or dug in. In this case, the fibre distribution hub only needs to provide the fibre cross-connection facility.

In both cases, the node or fibre distribution hub reverts to being completely passive reducing field maintenance and associated costs significantly. And, as previously, this conversion to FTTH/P can be undertaken progressively - either a node at a time or even by placing fibre distribution hub and MSAN side-by-side in the OSP and performing a gradual change over as customers are prepared to pay for FTTH/B enabled services. PTCL is the 
service provider company in Pakistan It has recognized the need for migrating its network infrastructure to NGN platform which can provide services efficiently and cost effectively to its customers. Thus the migration of PSTN has to be completed in least possible time and introducing new and advanced services efficiently so to construct a unified service platform to meet fixed and mobile customer's requirement in near future for optimal traffic and capacity for which the planning has been adopted and worked out in our paper.

\section{Capacity Planning in NGN}

The switching process is kept simple and the number of trunks would be fixed [6]. There is a serious level of dependence on soft switch for which if any problem arises there might be a large number of services interruptions and revenue lost besides the possibility of being punished by the authorities. NGN addresses the cost problem in addition to the deployment of network effectively. In order to distribute the traffic load evenly we look upon the scenario in terms of the signaling gateways, media resource server and the soft switch. As a first step we have to go for a proper location, the number of network components of NGN and the link capacity between NGN elements. We divide the legacy Network in domains thus deploying the softswitch and a signaling gateway in each domain. We are also aware that in the NGN system we would replace the class 4 switches by Trunk Gateway. Some operators are using the MSAN as an access gateway. After identifying the location and nodes the bandwidth has to be mentioned for the capacity calculation to ensure the timely availability of users in order to access the network.

The traffic intensity generated by each user is equal to call request completed per unit time multiplied by the holding time. The traffic intensity generated by each user in Erlangs is given by

$$
A_{u}=\lambda H
$$

$H$ is average duration of a call and $\lambda$ is average number of call requests per unit time for each subscriber. For a system having $U$ subscribers and unspecified number of channels, the total offered traffic intensity $A$, is given as $A=U A_{u}$.

In a $C$ channel trunked system, if traffic is equally distributed among the channels, then the traffic intensity per channel is denoted as $A_{c}$ which would be mathematically represented in the relation

$$
A_{c}=\frac{U A_{u}}{C}
$$

Link Capacity, LC1 = Bandwidth allocated amongst 2 Softswitches using SIP-T protocol.
Link Capacity, LC2 = Bandwidth allocated for traffic between Softswitch and Signaling

Gateway $=\lambda \times$ No. of Messages $/$ Call $\times$ No. of Bytes/Message.

Link Capacity, LC3 = Bandwidth allocated for Traffic between Softswitch and Media Resource Server $=\lambda \times$ No. of messages/Call $\times$ No. of Bytes/Message.

Link Capacity, LC4 $=$ Bandwidth allocated for Traffic between Softswitch and Trunk Gateway $=\lambda \times$ No. of messages/Call $\times$ No. of Bytes/Message.

Some service providers are using MSAN as Access Gateway so for traffic from Softswitch to MSAN.

Link Capacity, LC5 = Bandwidth allocated for Traffic between Softswitch and Access Gateway (or MSAN) = $\lambda \times$ No. of messages/Call $\times$ No. of Bytes/Message.

Link Capacity, LC6 = Bandwidth allocated for Traffic between Trunk Gateway and IP Core $=$ Total Number of Trunks $\times$ Average Traffic/Trunk $\times$ Payload Flow. The total traffic being A so LC6 $=\mathrm{A} \times$ Payload Flow.

Hence the overall capacity required between the trunk gateway and IP core network is dependent upon the number of trunks managed by each trunk gateway and the amount of traffic generated by the system subscribers.

\section{Conclusions}

NGN deployment has been kicked off and service providers are migrating their networks to NGN. The goal is to reduce costs of building and operating number of separate networks so we have emphasized on the softswitch to provide solution to migrate the PSTN to NGN. The network capacity planning is also dependent on the traffic distribution through softswitch.Voice services are now becoming less profitable for service providers so NGN based on IP is a way to bring the operating costs down thus contributing as a major driving factor for network operators. Operators would have to adopt IP due to huge efficiency, capacity and flexibility. So the service providers have to promote the delivery of "content" across the packet switched networks to continue with the way forward. Video on Demand offering voice, video and data is the most prioritized service to be provided to customers. The convergence of telecom and ip networks is the correct way to create the universal multimedia NGN. The existing network infrastructure cannot come up to the demands of the customers so operators have to provide up to $100 \mathrm{Mbps}$ and above to the subscribers' premises. Integrating all services on a single Multi Service Access Node would be more cost effective streamlining the way forward for provision of new services to customers as quick as possible. 


\section{References}

[1] M. Shakir, "Challenging Issues in NGN Implementation and Regulation," 2010 6th International Conference on Wireless Communications Networking and Mobile Computting, Chengdu, 23-25 September 2010, pp. 1-4. doi:10.1109/WICOM.2010.5600768

[2] ITU-T Recommendation Y2001, "General Overview of NGN,” December 2004.

[3] Sato, "Organising Innovation in Services: The Case of Telecommunications Next Generation Networks,” First ITU-T Kaleidoscope Academic Conference of Innovations in NGN: Future Network and Services, Geneva, 12-13
May 2008, pp. 255-262.

[4] N. Iiyama, H. Shiba, H. Kimura and H. Hadama, "Long term Cost-Effective Access Network for Fixed Mobile Convergence,” 2010 15th OptoeElectronics and Communications Conference, Sapporo, 5-9 July 2010, pp. 426427.

[5] C. Storbeck, "Next Generation Networks: The MSAN Strategy,” Fibresystems Europe Magazine, Boulder, June 2009.

[6] A. Amer, "Design and Capacity Planning of Next Generation Network (NGN)," Proceedings of the Mosharaka International Conference on Communications, Signals and Coding, Amman, 17-19 October 2008, pp. 28-34. 